\title{
Hemangioma in the Male Breast
}

\author{
Farhood Farid Mojtahedia, c, Tomas Bulut ${ }^{\mathrm{a}}$, Richard Oosterhof-Berktas ${ }^{\mathrm{b}}$, \\ Roland Bezooijen ${ }^{\mathrm{a}}$
}

\begin{abstract}
We saw a 52-year-old Caucasian male in our breast care clinic. The patient had noticed a small mass in his right breast for the past couple of months without any symptoms. After undergoing several diagnostic tests, the diagnosis was a breast hemangioma. This is a rare finding in male patients, and only a couple of cases have been described before.
\end{abstract}

Keywords: Hemangioma; Tumor; Mammography; Ultrasound; Biopsy; Benign

\section{Introduction}

We report a case of a male who presented in our clinic with a small mass in his right breast. Pathologic examination showed that the lesion was a breast hemangioma. A breast hemangioma is extremely rare in a male breast and has only been reported a few times before.

\section{Case Report}

A 52-year-old Caucasian male patient was admitted to our breast care clinic after noticing a small mass in his right breast which persisted for several months. His medical history included a thoracic aorta aneurysm and COPD, but no known malignancies or an increased risk for breast cancer.

A mammography was performed in a right cranio-caudal (RCC) and right medial-lateral oblique (RMLO) direction (Fig. 1). The obtained images revealed two round, circum-

Manuscript accepted for publication June 29, 2016

${ }^{a}$ Medisch Spectrum Twente, Koningsplein 1, 7512 KZ, Enschede, the Netherlands

${ }^{\text {b} M a r t i n i ~ Z i e k e n h u i s, ~ V a n ~ S w i e t e n p l e i n ~ 1, ~} 9728$ NT, Groningen, the Netherlands

${ }^{\mathrm{c}}$ Corresponding Author: Farhood Farid Mojtahedi, Reutumbrink 4, 7544 XL, Enschede, The Netherlands. Email: f.faridmojtahedi@mst.nl

doi: http://dx.doi.org/10.14740/jmc2578w scribed, high-density lesions in the breast.

The first lesion was located prepectorally in the upper outer quadrant.

The second lesion showed the same characteristics on mammography and was located in the lower outer quadrant. No anomalies were seen regarding the skin, areola or nipple.

An ultrasound of the right breast was performed for further detailed characteristics. The lesion in the upper outer quadrant measured approximately $11 \mathrm{~mm}$ in length and showed welldefined margins (Fig. 2). This lesion was mixed echoic with a hyper-echoic center and a hypo-echoic outer lining of 1.7 $\mathrm{mm}$ in thickness. Color Doppler ultrasound showed a single vascular pole. There was a horizontal orientation and no invasive features or distortion of the surrounding tissues. The mass showed typical characteristics of a lymph node with a fatty center and was therefore considered to be a BIRADS-2, according to the "Breast Imaging-Reporting and Data System" classification.

The lesion in the lower outer quadrant measured $7 \mathrm{~mm}$ in diameter and was also round and well-defined. Furthermore, it showed a mixed echo texture without invasive features or distortion of the surroundings in any way. No flow was seen on Doppler ultrasound (Fig. 2). Although the lesion showed benign morphologic characteristics, it did not show the typical benign features of male breast tissue. The lesion was considered to be a BIRADS-3 and therefore a core biopsy was performed.

The results of the histopathologic evaluation showed varying vascular structures with varying thin and thick walls, covered in flat endothelial cells; tissue was consistent with a hemangioma.

\section{Discussion}

A hemangioma is a very rare tumor in the male breast with an unknown incidence. Up until now, only large palpable masses have been reported as histological proven hemangiomas in male breasts.

A case of a hemangioma in the male breast has been reported four times before. The sizes of the hemangiomas varied from 4 to $51 \mathrm{~cm}$. In all cases, the patients presented themselves in the clinic with a palpable mass. Core biopsy showed endothelial lining. In some cases, where the hemangioma was big in size, a resection was performed [1-4]. 

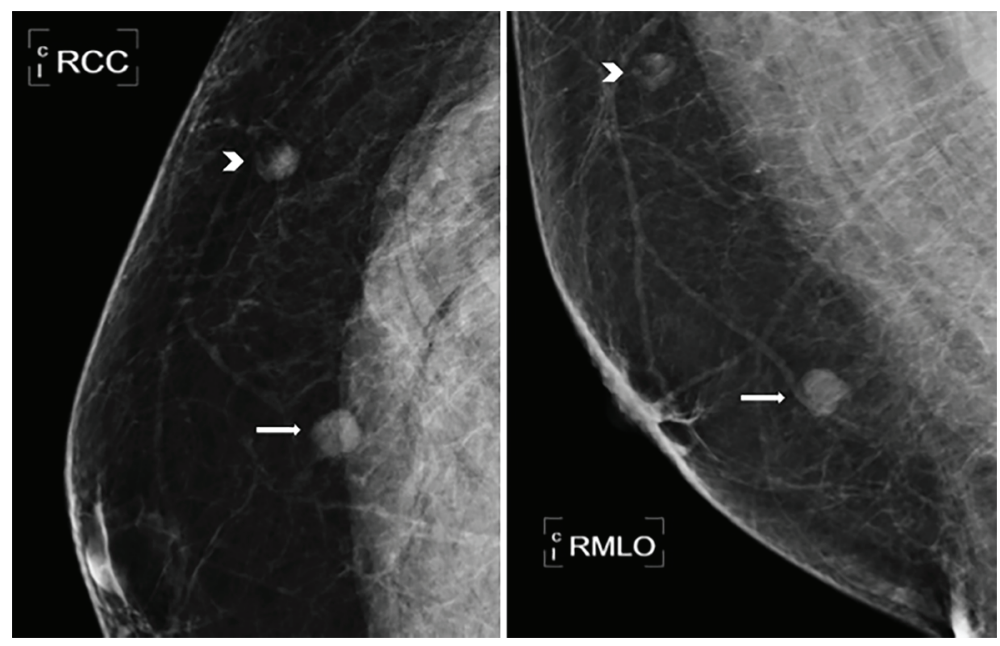

Figure 1. Mammogram of two well-defined lesions seen at 08:00 (arrow) and 11:00 (arrowhead).

As far as we could find in online scientific databases, these are the only reported cases of proven hemangiomas in the male breast.

In this case, we have reported the smallest hemangioma in a male breast up until now, measuring only $7 \mathrm{~mm}$. Unlike previously reported cases, lobulation was not a feature of this hemangioma.

Besides the lack of available pathognomonic characteristics on conventional imaging, the size of the hemangioma made diagnosis even more challenging.

\section{Conclusions}

Mammographically, hemangiomas appear as round or oval masses with sharp margins and may contain calcifications. Calcification is a known feature, but unspecific in order to distinguish from malignant lesions.

Sonography usually shows lobulated and well-circumscribed masses with variable echo patterns, mostly due to vascular channels and phleboliths. The surrounding tissue is usually not affected and drop shadow is not a typical feature. Unfortunately, sonographic characteristics are unspecific as well and make it difficult to distinguish between other benign lesions such as fibroadenoma, hematoma, arteriovenous mal- formations, venous malformations, lymphatic malformations or capillary malformations. Moreover, malignant tumors such as fibro-sarcoma and even angiosarcoma, should also be considered. They can show similar features on sonography, but Doppler might show multiple vascular poles suggesting vascularization.

The diagnosis of hemangioma is based on histopathologic analysis. Histologically angiosarcomas can present like hemangiomas; however, they show necrosis and hemorrhages on a frequent basis $[5,6]$.

If the tumor cannot be differentiated through histopathology, resection is recommended in case of doubt [3]. In our case, we have a histologically proven benign tumor, therefore resection was not necessary.

\section{Grant}

This research did not receive any specific grant from funding agencies in the public, commercial, or not-for-profit sectors.

\section{Conflicts of Interest}

None.
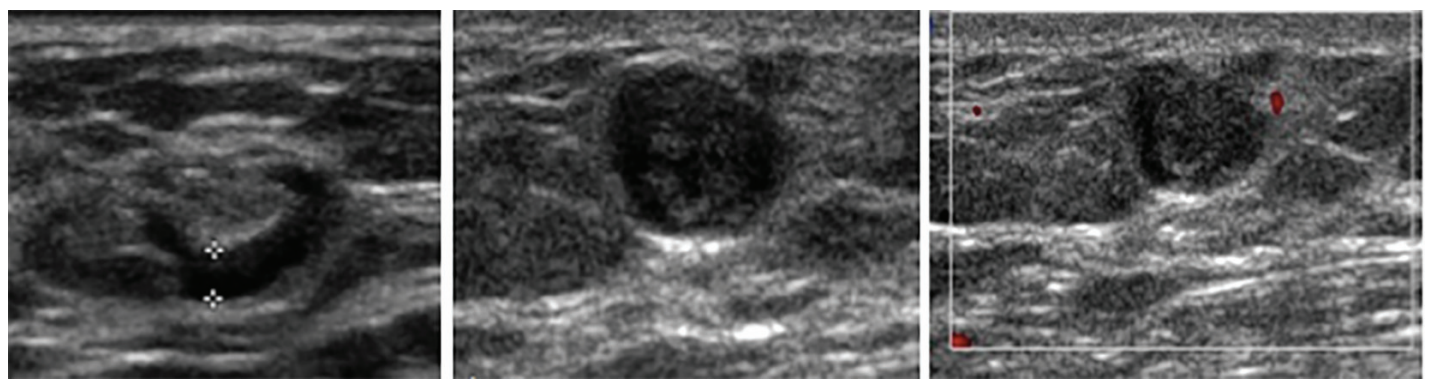

Figure 2. Ultrasound of a well-defined oval-shaped lesion with a fatty center and hypo-echoic outer lining of $1.7 \mathrm{~mm}$ in thickness at 11:00. Lesion was considered to be a non-pathological lymph node, BI-RADS2 (left). A well-defined hypo-echoic lesion with hyper-echoic focal central areas was seen at 08:00, but without vascularization, BIRADS 3 (middle and right). 


\section{References}

1. Smythe FW. Brief communication: intramammary hemangioma. Annals of Surgery, 1942, 115th edition, p.716-719.

2. Kinoshita S, Kyoda S, Tsuboi K, Son K, Usuba T, Nakasato $\mathrm{Y}$, Kashiwagi $\mathrm{H}$, et al. Huge cavernous hemangioma arising in a male breast. Breast Cancer. 2005;12(3):231233.

3. Coronel-Brizio P, Uscanga Chavez E, Noguera Martinez JL, Libreros Morales EA. [Cavernous hemangioma of the breast]. Ginecol Obstet Mex. 2003;71:527-531.

4. Vourtsi A, Zervoudis S, Pafiti A, Athanasiadis S. Male breast hemangioma--a rare entity: a case report and review of the literature. Breast J. 2006;12(3):260-262.

5. Tilve A, Mallo R, Perez A, Santiago P. Breast hemangiomas: correlation between imaging and pathologic findings. J Clin Ultrasound. 2012;40(8):512-517.

6. Leddy R, Cluver A. Mammographic and sonographic characteristics of a cavernous hemangioma in a male patient. J Ultrasound Med. 2010;29(4):645-649. 